to vancomycin ( $\mathrm{MIC}=8 \mu / \mathrm{mL}$ ); all previous MRSA strains had been vancomycin-susceptible. This VISA isolate was sent to the CDC, where the intermediate resistance was confirmed; the isolate was susceptible to gentamicin, trimethoprim-sulfamethoxazole, tetracycline, and imipenem. The patient continues to receive antimicrobial therapy at home.-ED.]

FROM: 1. Centers for Disease Control and Prevention. Reduced susceptibility of Staphylococcus aureus to vancomycin-Japan, 1996. MMWR 1997;46:624-626.

2. Centers for Disease Control and Prevention. Staphylococcus aureus with reduced susceptibility to vancomycin-United States, 1997. MMWR 1997;46:765-776.

3. Centers for Disease Control and Prevention. Interim guidelines for prevention and control of staphylococcal infection associated with reduced susceptibility to vancomycin. MMWR 1997;46:626-628,635.

\section{Vancomycin Resistance Outside the Healthcare Setting}

Although no data so far support substantial acquisition and transmission of vancomycin-resistant enterococci (VRE) outside the healthcare setting in the United States, a growing number of reports from Europe suggest that colonization with VRE occurs frequently in the community. Reports from Europe also have suggested that VRE exist elsewhere in the environment, including animal feces and human foods of animal origin. Additional evidence supports the transmission of VRE to persons in contact with these sources, resulting in an increased human reservoir of VRE colonization.

An important factor associated with VRE in the community in Europe has been avoparcin, a glycopeptide antimicrobial drug used for years in many European nations at subtherapeutic doses as a growth promoter in food-producing animals. Although avoparcin never has been approved for use in the United States, undetected community VRE transmission may be occurring at low levels. Further studies of community transmission of VRE in the United States are needed urgently. If transmission with VRE from unrecognized community sources can be identified and controlled, increased incidence of colonization and infection among hospitalized patients may be prevented.

FROM: McDonald LC, Kuehnert MJ, Tenover FC, Jarvis WR. Vancomycin-resistant enterococci outside the health-care setting: prevalence, sources, and public health implications. Emerg Infect Dis 1997;3:311-315.

\section{Electroconvulsive Therapy-Related Bacteremia}

Infectious complications associated with electroconvulsive therapy (ECT) are extremely unusual. When five of nine patients undergoing ECT at one facility on June 20, 1996, developed Staphylococcus aureus bloodstream infection
(BSI), an investigation by the CDC's Hospital Infections Program was initiated.

A case was defined as any patient who had ECT at facility A from June 1, 1995, through June 20, 1996, and developed $S$ aureus BSI less than 30 days after ECT. The post-ECT $S$ aureus BSI rate was significantly greater on the epidemic day than the pre-epidemic period, (ie, June 1, $1995-J u n e \quad 19,1996 ; 5 / 9$ vs $0 / 54$ patients, $P<.001)$. All patients during the study period received propofol before ECT. Case patients were more likely than non-case patients to have higher maximum temperature after ECT (median, $103.9^{\circ} \mathrm{F}$ vs $\left.100.0^{\circ} \mathrm{F} ; P<.03\right)$ and a greater time from preparation of intravenous medications to infusion (median, $2.1 \mathrm{vs}$ 1.1 hours; $P=.01$ ). All isolates of $S$ aureus from case patients were indistinguishable by pulsed-field gel electrophoresis. These data suggest the ECT-associated $S$ aureus BSIs were associated with the administration of propofol that was contaminated during preparation due to multiple breaks in aseptic technique.

FROM: Kuehnert MJ, Webb RM, Jochimsen EM, et al. Staphylococcus aureus bloodstream infections among patients undergoing electroconvulsive therapy traced to breaks in infection control and possible extrinsic contamination by propofol. Anesth Analg 1997;85:420-425.

\section{Compliance With OSHA's Ethylene Oxide Standard}

Researchers in Massachusetts conducted a study to determine the extent to which hospitals in the state implemented the Occupational Safety and Health Administration (OSHA)'s 1984 ethylene oxide (EtO) standard. EtO is used in hospitals to sterilize heat- and moisture-sensitive medical devices and instruments. Healthcare workers comprise the largest group among the estimated 270,000 US workers who are potentially exposed to EtO. OSHA considers EtO a potent neurotoxin, a known human carcinogen, a potential reproductive hazard, and an allergic sensitizer.

In 1984, OSHA published a health standard setting at $1 \mathrm{ppm}$ permissible exposure limit (PEL) and $0.5 \mathrm{ppm}$ action level. The standard was revised in 1988 to add a 5 ppm short-term excursion limit. The EtO standard requires exposure monitoring consisting of workers' breathing zone air samples that are representative of the 8-hour timeweighted average (for PEL and action level) and 15-minute short-term exposures for each employee (for excursion limit). If exposures exceed that action level or excursion limits, repeat testing is required.

An in-depth mail and telephone survey was conducted followed by on-site interviews at all EtO-using hospitals in Massachusetts ( $n=92 ; 96 \%$ participation rate). The study results showed that, by 1993, most hospitals had performed personal exposure monitoring for OSHA's 8-hour action level (95\%) and the excursion limit (87\%), although most did not meet the 1985 implementation deadline. In 1993, 66\% of hospitals reported the installation of EtO alarms to fulfill the standard's "alert" requirement. Alarm 